\title{
Genomic analysis of Campylobacter fetus subspecies: identification of candidate virulence determinants and diagnostic assay targets
} Paula M Moolhuijzen* ${ }^{* 1}$, Ala E Lew-Tabor ${ }^{\dagger 1,2}$, Bartosz M Wlodek ${ }^{2}$, Fernán G Agüero ${ }^{3}$, Diego J Comerci ${ }^{3}$, Rodolfo A Ugalde ${ }^{3}$, Daniel O Sanchez ${ }^{3}$, Rudi Appels ${ }^{1}$ and Matthew Bellgard ${ }^{1}$

\author{
Address: ${ }^{1}$ Centre for Comparative Genomics, School for Information Technology, Murdoch University, Murdoch, Western Australia, Australia, \\ ${ }^{2}$ Emerging Technologies, Queensland Department of Primary Industries \& Fisheries, Yeeongpilly, Queensland, Australia and ${ }^{3}$ Instituto de \\ Investigaciones Biotecnológicas, Universidad Nacional de General San Martín - CONICET, 1650 KNA, San Martín, Buenos Aires, Argentina \\ Email: Paula M Moolhuijzen* - pmoolhuijzen@ccg.murdoch.edu.au; Ala E Lew-Tabor - Ala.Lew@dpi.qld.gov.au; \\ Bartosz M Wlodek - Bartosz.Wlodek@dpi.qld.gov.au; Fernán G Agüero - fernan@iib.unsam.edu.ar; \\ Diego J Comerci - dcomerci@iib.unsam.edu.ar; Rodolfo A Ugalde - rugalde@iib.unsam.edu.ar; Daniel O Sanchez - dsanchez@unsam.edu.ar; \\ Rudi Appels - rappels@ccg.murdoch.edu.au; Matthew Bellgard - mbellgard@ccg.murdoch.edu.au \\ * Corresponding author †Equal contributors
}

Published: 8 May 2009

BMC Microbiology 2009, 9:86 doi:10.1186/147|-2180-9-86
Received: 9 October 2008

Accepted: 8 May 2009

This article is available from: http://www.biomedcentral.com/I47I-2/80/9/86

(C) 2009 Moolhuijzen et al; licensee BioMed Central Ltd.

This is an Open Access article distributed under the terms of the Creative Commons Attribution License (http://creativecommons.org/licenses/by/2.0), which permits unrestricted use, distribution, and reproduction in any medium, provided the original work is properly cited.

\begin{abstract}
Background: Campylobacter fetus subspecies venerealis is the causative agent of bovine genital campylobacteriosis, asymptomatic in bulls the disease is spread to female cattle causing extensive reproductive loss. The microbiological and molecular differentiation of $C$. fetus subsp. venerealis from $C$. fetus subsp. fetus is extremely difficult. This study describes the analysis of the available $C$. fetus subsp. venerealis AZUL-94 strain genome $(\sim 75-80 \%)$ to identify elements exclusively found in $C$. fetus subsp. venerealis strains as potential diagnostic targets and the characterisation of subspecies virulence genes.

Results: Eighty Kb of genomic sequence (22 contigs) was identified as unique to $C$. fetus subsp. venerealis AZUL-94 and consisted of type IV secretory pathway components, putative plasmid genes and hypothetical proteins. Of the 9 PCR assays developed to target $C$. fetus subsp. venerealis type IV secretion system genes, 4 of these were specific for $C$. fetus subsp. venerealis biovar venerealis and did not detect $C$. fetus subsp. venerealis biovar intermedius. Two assays were specific for $C$. fetus subsp. venerealis AZUL-94 strain, with a further single assay specific for the AZUL-94 strain and C. fetus subsp. venerealis biovar intermedius (and not the remaining $C$. fetus subsp. venerealis biovar venerealis strains tested). C. fetus subsp. fetus and $C$. fetus subsp. venerealis were found to share most common Campylobacter virulence factors such as SAP, chemotaxis, flagellar biosynthesis, 2-component systems and cytolethal distending toxin subunits $(A, B, C)$. We did not however, identify in $C$. fetus the full complement of bacterial adherence candidates commonly found in other Campylobacter spp.

Conclusion: The comparison of the available $C$. fetus subsp. venerealis genome sequence with the $C$. fetus subsp. fetus genome identified $80 \mathrm{~kb}$ of unique $C$. fetus subsp. venerealis AZUL94 sequence, with subsequent PCR confirmation demonstrating inconsistent amplification of these targets in all other $C$. fetus subsp. venerealis strains and biovars tested. The assays developed here highlight the complexity of targeting strain specific virulence genes for field studies for the molecular identification and epidemiology of $C$. fetus.
\end{abstract}




\section{Background}

Northern Australian beef herds have a 35\% unexplained reduction in calf production. In Argentina, calf production has not declined, but remains at a constantly low rate (63\%). To aid the detection and treatment of cattle infected with Campylobacter fetus our genomic analysis has identified candidate subspecies specific genes that can be used as diagnostic tools.

The Campylobacter genus is a Gram-negative, spiral-shaped bacterium and includes 23 recorded species in the NCBI Taxonomy division. Campylobacter spp. colonise diverse hosts from livestock to humans with varying degrees of virulence [1]. Hosts include cattle, swine, bird, and can be the major cause of human bacterial gastroenteritis [2]. $C$. fetus subsp. venerealis ( $C f v$ ) is the causative agent of bovine genital campylobacteriosis, which causes conception failure and embryo loss, with bulls acting as asymptomatic carriers [3]. C. fetus subsp. fetus (Cff) causes infertility and infectious abortions in domesticated sheep, goats and cattle [2]. It is also an opportunistic pathogen in humans that can severely affect immuno-compromised patients. Initially the bacterium can cause gastroenteritis, and then spread systemically throughout the blood (bacteremia) and cause septicaemia, meningitis, and other systemic infections [2]. Bovine genital campylobacteriosis is an Office International des Epizooties (OIE) notifiable disease considered to have socio-economic and public health implications, particularly with respect to the international trade of animals and animal products [4].

Although Campylobacter sub species have largely conserved genomes, sub species display variable virulence phenotypes in animal models and this phenotypic virulence has been speculated to be due to hyper-variable antigenic diversity and immune evasion $[1,5]$. Very few gene targets have been identified for the differentiation of $C$. fetus subspecies, with members of the subspecies shown to be $86 \%$ similar based on PFGE-DNA profiles [6]. Diagnostic testing of $C$. fetus colonies from transport medium and the biochemical differentiation of the 2 subspecies venerealis and fetus is important for the diagnosis of bovine venereal disease in cattle. $C f f$ and $C f v$ can be differentiated from each other using a range of biochemical assays including $\mathrm{H}_{2} \mathrm{~S}$, selenite reduction, growth at $42^{\circ} \mathrm{C}$, susceptibility to metronidazole and cefoperazone, basic fuchsin, $\mathrm{KMnO}_{4}$ and glycine tolerance [6,7]. Glycine tolerance is the OIE recommended assay. It is however difficult to isolate viable colonies from transport medium for biochemical analysis due to prolonged transport, contaminant overgrowth and the fastidious nature of the bacteria [8-10]. In addition doubts in regard to the stability of these biochemical markers has been suggested based on evidence from phage transduction [6,11-13]. The $\mathrm{H}_{2} \mathrm{~S}$ test although described as differentiating Cff (positive) and $C f v$ (negative), a $C f v$ strain subsequently named $C f v$ biovar intermedius is positive in this assay [14]. Molecular typing methods such as amplified fragment length polymorphism (AFLP) and multilocus sequence typing have been developed to differentiate $C$. fetus isolates $[11,15]$, but these methods require the isolation of pure colonies which are impractical for diagnostic application. Specific polymerase chain reaction (PCR) assays have been designed and applied to detect $C f v$ [16-18], however it has been suggested that the gene targets are plasmid borne and that in some cases have not reliably detected all $C f v$ isolates [19]. A sensitive real time assay designed to target the parA gene originally targeted by the Hum et al (1997) PCR assay, identified a high prevalence of Cfv in Australia cattle not associated with venereal cases [4]. It was thus postulated that isolates of $C f v$ differ in virulence and that other methods may be required to confirm the presence of pathogenic $C f v$ in clinical samples. Genomic Campylobacter comparisons of C. fetus subspecies and a list of $C f v$ specific genes will provide the basis for developing specific diagnostic assays and improving our understanding of C. fetus virulence and epidemiology. No studies to date have reported the putative identification or extensive analysis of $C f v$ virulence genes.

Based on comparative analysis on recently available genome data for both $C$. fetus subsp. venerealis ( $C f v$ ) (incomplete) and C. fetus subsp. fetus (Cff) we have developed a number of assays targeting virulence factors previously identified in C. jejuni, C. coli, C. lari, and C. upsaliensis genomes. These virulence mechanisms include motility, chemotaxis, adhesion, invasion and toxin production and regulation by two-component systems, as discussed in Fouts et al [1]. This paper provides the first detailed analysis of available genome sequences in order to identify targets for differentiating C. fetus subspecies. Based on the analysis several targets were identified and confirmed using PCR assays.

Our aims were to (1) identify and compare C. fetus putative virulence genes, (2) characterise genomic features to differentiate the highly conserved C. fetus subspecies for diagnostic assays. The genomic features of Campylobacter provided subspecies markers that discriminate C. fetus species and subspecies, in particular the C. fetus sub species (Cfv and Cff) from each other and other Campylobacter species.

\section{Results}

\section{Assembly of Cfv for Identifying Targets for Diagnostics}

The available genomic sequence information (ca 75-80\% Cfv genome) was compiled using the complete Cff 82-40 genome sequence (NC_008599) in order to identify tar- 
gets for the diagnostics for detecting $C f v$. The ordering of available genome segments generally aligned well with the Cff genome as shown in Figure 1.

Several striking features were evident in the subspecies comparison. Firstly, an $80 \mathrm{~Kb}$ suite of $22 \mathrm{Cfv}$ specific contigs (relative to $\mathrm{Cff}$ ) housed a range of putative virulence factors such as Type IV secretion systems (Additional file 1 ). Secondly a number of potential virulence factors were also identified in the genomic sequences that were shared between Cfv and Cff (Additional file 2). Table 1 summarises virulence factors by comparing the ORFs of the $2 \mathrm{C}$. fetus subspecies with 4 Campylobacter species as described in Fouts et al (2005). In general similar numbers of genes potentially associated with 2 component systems, toxin production, outer membrane proteins, and motility were identified. Only one bacterial adherence gene was identified in both C. fetus subspecies with 2 and 3 ORFs identified in Cfv and Cff respectively (Table 1). However an additional adhesion homologue fibronectin (FN) binding
ORF was identified in $C f v$ but not $C f f$. A large surface array protein was found highly conserved in both species (not shown in this study) but was evident in the genomic sequence alignments (figure 1 ).

The nucleotide alignment of Cfv contigs based on the closest sequenced genome Cff displayed the Cfv contig sequence in common between the two genomes (not specific to Cfv) and Cfv contig sequence not found in Cff (specific to Cfv) (Figure 1). Of the $273 \mathrm{Cfv}$ contigs, 251 contigs ( $993569 \mathrm{bp}$ ) were conserved with Cff and 22 contigs (86999 bp) specific to the Cfv genome compared to Cff. Contigs specific to Cfv were Contig1018, Contig1021, Contig1023, Contig1024, Contig1030, Contig1031, Contig1042, Contig1120, Contig1139, Contig1165, Contig1181, Contig1185, Contig1186, Contig419, Contig733, Contig846, Contig851, Contig872, Contig875, Contig914, Contig958 and Contig991 (ORF without strong homology to Cff are listed in Additional file 1).

\subsection{Mb C. fetus subsp. venerealis (Cfv) contigs}

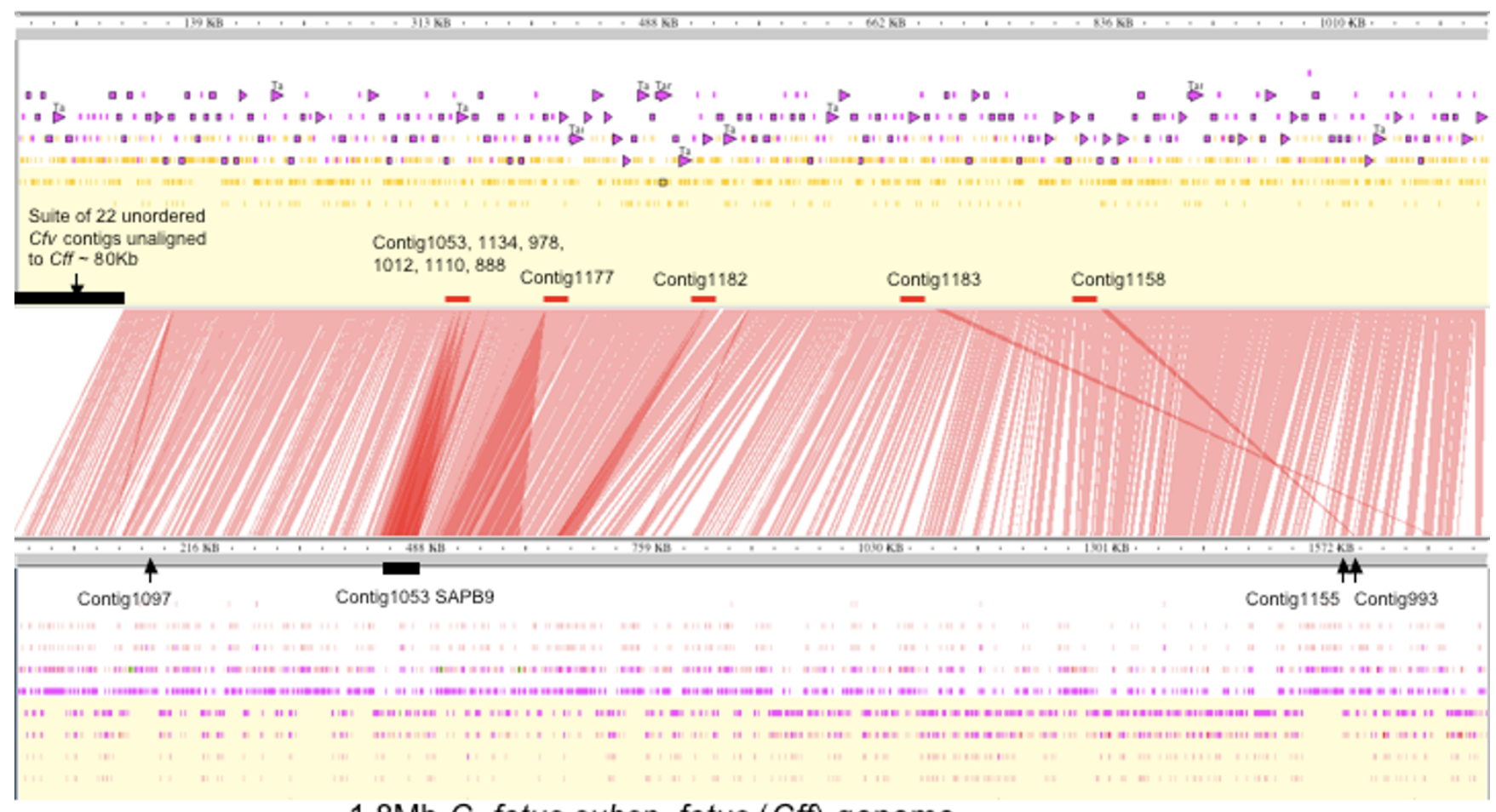

1.8Mb C. fetus subsp. fetus (Cff) genome

\section{Figure I}

Genomic nucleotide alignment of $C$. fetus subsp. venerealis $(C f v)$ contigs to the $C$. fetus subsp. fetus genome. Genomic nucleotide comparison of $C$. fetus subsp. venerealis ( $C f v)$ contigs (I.08 Mb) as aligned to the $C$. fetus subsp. fetus (Cff) completed genome (I.8 Mb). Orange shaded regions between the parallel sequences of $C f v$ (top) and Cff (bottom) highlight contigs in common and unique between the two Campylobacter subspecies. 
Table I: $C$. fetus subsp. fetus ( $C \mathrm{ff}$ ) and subsp. venerealis ( $C f v)$ virulence factors compared with 4 other Campylobacter spp.

\begin{tabular}{llll}
\hline Putative virulence type & Other spp. $^{\text {a }}$ & Cff & $C_{f v^{*}}$ \\
\hline Bacterial adherence & 9 & $3 \mathrm{~b}$ & $4 \mathrm{~b}$ \\
\hline Motility & $55-66$ & 41 & 46 \\
\hline Two-component system genes & $11-15$ & 16 & 14 \\
\hline Toxin and resistance & $15-20$ & $9 c$ & $7 \mathrm{c}$ \\
\hline Membrane proteins & $185-218$ & 209 & 202 \\
\hline
\end{tabular}

Summary of $C$. fetus virulence gene ORFs in $C$. fetus subsp. fetus ( $C f f)$ and subsp. venerealis ( $C f v)$ compared with 4 other Campylobacter spp. (adapted from Fouts et al).

a C. jejuni, C. lari, C. upsaliensis, C. coli (Fouts et al. 2005)

${ }^{b} C f f-P E B I$ (3) - no other adherence homologues found; C $F V$ ORFs -

PEBI (2), cadF(0), jlpA (I-poor homology), Fibronectin binding (I), 43kDA MOMP (0)

${ }^{c}$ not including resistance genes for $C f f$ and $C f v$, toxin subunit ORFs only

*N.B. Cfv genome incomplete

When probed against all available genome protein sequence information the Cfv specific contigs (Additional file 3: Table S1) had the following alignments; two contigs $(\sim 4.9 \mathrm{~Kb})$ with short alignments to only non-campylobacter bacterial species (Contigs914 and 875) (Campylobacter specific); five contigs $(\sim 20 \mathrm{~Kb})$ with significant alignments to $C$. jejuni and C. coli plasmid genomes and short alignments to $C$. hominis and $C$. lari; ten contigs completely unique to $C f v$ (Cfv specific) ( $\sim 32 \mathrm{~Kb})$; and five contigs $(\sim 27 \mathrm{~Kb})$ with significant protein alignments to Cff although this was not evident at the nucleotide sequence level.

\section{Cfv Open Reading Frame Analysis}

The C. fetus subsp. venerealis 1474 ORFs protein database search found 67 unique to $C f v$ (no protein alignments), 1174 conserved top match alignment to Cff, 116 conserved top match alignment to any other species, and 117 low significance alignments. ORF alignments to the nonredundant protein database found $12 \%$ Cfv insignificant and unique (Additional file 1), 51\% with significant alignments and $37 \%$ with highly significant alignments. Comparison of the 9 Campylobacter genome protein datasets found approximately $50 \%$ of proteins were in common for all C. jejuni (including subsp. jejuni and doyley) except C. jejuni subsp. jejuni 81-116 which had 20-25\% similar. This level of similarity was also found between the Cff subspecies while between all Campylobacter species this similarity decreased to between $0.5-5.5 \%$. The BlastMatrix [20] result can be found in additional file 4 .

\section{Cfv Open Reading Frame Analysis of the Cfv specific suite of genomic regions}

Eighteen $C f v$ specific contig ORFs were analysed against all available protein datasets. These $C f v$ specific regions contained 90 ORFs, 15 with alignments to hypothetical proteins, 32 with non-significant protein alignments and 43 ORFs with significant alignments. As a separate category these latter 43 ORFs were found to have significant alignments to plasmid/phage like proteins within Campylobacter species (34 ORFs) and to other bacteria (9 ORFs). In the 34 Campylobacter ORFs, best matches were found in two Cfv ORFs, namely a putative type IV secretion system protein identified in IsCfe 1 [18] and a putative TrbL/VirB6 plasmid conjugal transfer protein. The remaining $32 \mathrm{ORFs}$ had significant hits to Campylobacter species other than Cfv such as C. curvus (1), C. concisus (2), C. coli (4), C. fetus (5), C. jejuni (13) and C. hominis (17).

Functional assignments for the Cfv specific ORFs were as follows; cellular processes and signalling, chromosome partitioning, cell motility and intracellular trafficking, secretion and vesicular transport (16); information storage and processing, replication, recombination and repair, transcription, translation (12); metabolism and transport amino acid, carbohydrate and inorganic ion, energy production and conversion (5); and poorly characterized, general function prediction only (7) (Additional file 1).

\section{Cfv ISCfe I insertion elements}

Specific sites previously identified for the ISCfe 1 insertion element [18] were searched in Cfv alignments to Cff (Figure 1): (a) the sodium/hydrogen exchanger protein gene nahE (YP_891382) was found in the Cfv pseudomolecule positioned 159601-160764 bp (Contig1097), a region conserved with Cff; (b) the putative methyltransferase protein gene metT (YP_892765) was found in the Cfv pseudomolecule positioned 1605092-1603530 bp (Contig1155) a region also conserved in Cff; and (c) the putative VirB6 protein gene was found in a number of $C f v$ contigs, these include contigs with ORFs not common with Cff Contig1023 and Cfv specific Contig1165, Contig733, Contig875 and Contig958.

$C f v$ contigs were searched for the ISCfe 1 insertion containing sequences (AM260752, AM286430, AM286431 and AM286432). All the ISCfe1 sequences aligned to Contig993 (39-1464 bp) with greater than 90 percent identity. Contig993 Cff position is indicated in figure 1. The ISCfe 1 genes $\operatorname{tn} p A$ and $\operatorname{tn} p B$ matched Contig993 orf1 partial transposase A (Cfv) (14-157) and Contig993 orf2 transposase B (Cfv) (144-1436). Upstream ORF regions in Contig993 are: Contig993 orf3 anaerobic C4-dicarbox- 
ylate transporter (Cff) 1509-1697 bp; Contig993 orf4, anaerobic C4-dicarboxylate transporter (Cff) 1705-2493 bp; and Contig993 orf6 which had no protein alignments 2795-2968 bp.

Contig875 only aligned with AM286432 (21-1235 bp) putative virulence genes with $>90 \%$ sequence identity. Contig875 orf3 (499-1068 bp) partially to the partial putative virulence gene VirB5 and Contig875 orf5 (1302$2069 \mathrm{bp}$ ) to the truncated putative TrbL/VirB6 plasmid conjugal transfer $(C f v)$ gene. Downstream in Contig875 were Contig875 orf1 transposase OrfA (Helicobacter pylori) 30-170 bp and Contig875 orf2 (274-489 bp) with no protein alignments.

\section{Genomic Plasmid Analysis}

Plasmid containing Campylobacters include C. coli, C. lari, C. concisus 13826 (2 plasmids), C. hominis ATCC BAA-381 (1 plasmid), C. jejuni subsp. jejuni 81-176 (2 plasmids) and C. fetus subsp venerealis strain 4111/108. Complete plasmids have been sequenced for C. coli (6), C. lari (2), other C. jejuni strains (6) and C. fetus subsp venerealis (1). A direct search of these extrachromosomal Campylobacter plasmid sequences against $C f v$ specific sequence determined plasmid borne genes in common between the species. Plasmid sequences from C. coli, C. hominus and C. jejuni represent over a third of the Cfv specific ORFs (37/ 90). These include type IV secretion system (Vir and Cmg), ParA, Ssb, RepE, moblization and plasmid (Cpp and pTet) proteins (Additional file 3: Table S2). Tranposase genes were absent in the other Campylobacter spp. plasmids and found in Cfv Contigs1185 (2), Contig872 (1) and Contig875 (1). The C. fetus subsp venerealis plasmid $p C F V 108$ (EF050075) contains four genes, putative $m o b C$, putative mobA, repE and an uncharacterised orf3 [21]. Plasmid pCFV108 ws not found in the Cfv contigs. A protein search however found significant alignments for Contig1185.orf00004 to MobA (ABK41363 489 aa) and Contig1185.orf00007 to RepE (ABK41364 351 aa) (Additional file 5)

\section{COG Analysis -Virulence Genes}

The String database analyses identified $1141 \mathrm{Cfv}$ ORFs that aligned significantly to String assigned COG functions. Comparative analysis between $C f v$ to the Cluster Orthologous groups found $273 \mathrm{ORF}$ in cellular processing and signalling a COG role known to contain virulence determinants, 164 information storage and processing, 406 metabolism, 153 poorly characterised, 87 to hypothetical proteins and the remaining without assignments to COG roles.

COG role distributions for virulence ORFs can be found in additional file 2 .
In putative virulence roles, $49 \mathrm{Cfv}$ ORFs are involved in cell motility, 83 in cell wall/membrane/envelope biogenesis, 21 defence mechanisms, 25 intracellular trafficking, secretion and vesicular transport and 29 signal transduction mechanisms.

To identify virulence genes unique to $C f v$ or other Campylobacter species and distinguish the two subspecies, the Cff and $C f v$ virulence genes and $C f v$ contigs were aligned to the Cff genome. The non-redundant protein search also identified virulence genes such as Cytolethal distending toxin proteins (Cdt) [1], currently characterised as a hypothetical protein within the non-supervised orthologous groupings of String, although characterized and reported by Asakura et al in 2007 [22,23].

Based on COG analyses (Additional files 1 \&2) the following sequences found in categories that were both specific and not specific to $C f v$, were selected for PCR validation. Those selected for PCR included virulence genes (including the Type IV secretion genes specific to Cfv) (8), flagella (6), cytolethal distending toxin (3), response regulatorsensor (6), membrane (4), fibronectin (1), haemolysin (1), Fe ABC transporter (1) and mannose-1-phosphate guanylyltransferase/mannose-6-phosphate isomerase (1) genes (Additional file 3: Table S3).

\section{PCR Results}

To validate the subspecies specificity of virulence genes and $C f v$ specific sequences identified above, 31Cfv ORF sequences were selected in $C f v$ and primer sets tested using Cff and Cfv isolates (Additional file 3: Table S3). Reference and type strains screened are described in Table 2 and $C f v$ reference strains included $4 C f v$ biovar venerealis isolates (DPI, ATCC, UNSAM and Pfizer) and a Cfv biovar intermedius (Pfizer) isolate. Cff strains used were DPI and ATCC isolates as described in Table 2. All primers were based on the Cfv biovar venerealis AZUL-94 strain contig sequences except for flhA and flhB which were based on $C f f$ sequence for these 2 flagella genes not identified in Cfv contigs. Conserved amplification of virulence genes in both C. fetus subspecies included flagella, outer membrane proteins, 2 component systems (response regulators and sensors), haemolysin, iron uptake and a fibronectin type III domain protein (Additional file 3: Table S3). For assays based on ORFs selected as absent in Cff, contigs 1120 orf 4,1165 orfs 4,8 and 875 orf5 assays amplified the $C f v$ biovar venerealis strains but not $C f v$ biovar intermedius or the Cff reference strains. These contigs were identified as: VirB4, VirB11, VirD4 and VirB6 type IV secretion system proteins respectively. Three assays (1023 orf2/VirB10, 1023 orf3/VirB11 and 733 orf1/VirB4) were specific for Cfv biovar venerealis AZUL-94 strain and did not amplify other biovar venerealis strains. One of these 
assays Contig 1023 orf 3 (VirB11) also amplified Cfv biovar intermedius. Cfv biovar intermedius was negative in all other ' $C f v$ ' specific assays, which in the current study appear to be specific for $C f v$ biovar venerealis. Curiously, 1 assay based on 1165 orf 2 (Cfv VirB9) was positive for $C f v$ biovar venerealis AZUL-94, Cfv biovar intermedius and both Cff strains tested but did not amplify the other $3 \mathrm{Cfv}$ biovar venerealis strains including the ATCC 19438 strain. All assays were specific for $C$. fetus subspecies, testing negative in related strains and reproductive disease pathogens listed in Table 2 including: C. coli, C. jejuni, C. sputorum subsp. bubulus, C. hyointestinalis, Pseudomonas aeruginosa, Proteus vulgaris, Neospora caninum and Tritrichomonas foetus (results not presented). However no single assay amplified all $C f v$ strains inclusive of both biovars venerealis and intermedius. Figure 2 demonstrates the specificity of selected primer sets Contig1023 orf2 and orf3, Contig1154 orf3 and Contig1165 orf4. Contig1023 orf3 and Contig1165 orf4 primers amplified sequences specific for $C f v$, while Contig1154 orf3 primers amplified sequences in both $C f v$ and $C f f$ strains.

\section{Discussion}

The available $C f v$ genomic sequence information was aligned to the complete Cff genome sequence 82-40 in order to identify targets for the diagnostics for detecting $C f v$. Based on the genome size estimates of $C f v[6,24]$ and

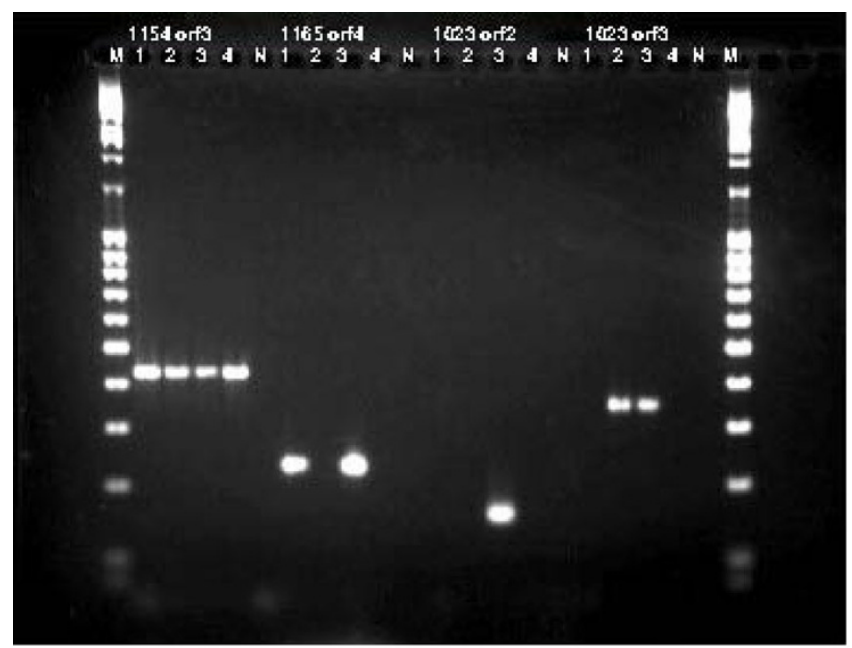

Figure 2

PCR assay specificity for $C$. fetus subspecies and $C$. fetus subsp veneralis. Examples of $P C R$ assay specificity for $C$. fetus subspecies and $C$. fetus subsp veneralis biovars (venerealis and intermedius). Lanes numbered $\mathrm{I}-4, \mathrm{~N}$ and $M$ represent: I Cfv biovar venerealis 19438 ATCC, 2 Cfv biovar intermedius (Pfizer strain), 3 Cfv Argentina AZUL-94 strain, 4 Cff I5296 ATCC, $\mathrm{N}=$ negative no template control and $M=$ molecular weight marker 100 bp ladder (Invitrogen). Results are shown for assays based on Contig I I 54 orf3 (429 bp), Contig I I 65 orf4 (233 bp), Contig 1023 orf2 (I59 bp) and Contigl 023 orf3 (349 bp). the completed Cff genome size, it is estimated that approximately $72 \%$ of the $C f v$ genome has been sequenced (unpublished, Prof Daniel Sanchez, Universidad Nacional de San Martin, Argentina). The ordering of available genome segments generally aligned well with the Cff genome as shown in Figure 1 and made evident a suite of Cfv specific contigs. This suite of contigs housed a large range of type IV secretion factors, and plasmid/ phage like proteins. A number of potential virulence factors were clearly identified as shared between $C f v$ and $C f f$. These virulence factors include an outer surface array membrane protein, chemotaxis types, motility associated, regulatory and secretion systems. The existence of these classes of genes with roles in the infection process, but not showing sub species specificity, is consistent with a twotier infection model. Surface/membrane components provide necessary (but not sufficient) structural components for attachment to host cells. Specific components that complete the features of the surface/membrane structures are required for infection. Fouts et al., (2005) found that many genes involved in host colonization were conserved across the Campylobacter genus. Variations that were species specific were evident for a lipo-oligosaccharide locus, a capsular (extracellular) polysaccharide locus, and a novel Campylobacter putative licABCD virulence locus (not found in available Cfv). These observations are consistent with the suggestions that interactions between a pathogen's surface-exposed proteins and host cells represent a pivotal step in pathogenesis and virulence [25]. In pathogens several of the key players are proteins involved in adhesion, invasion, secretion, signalling, annulling host responses, toxicity, motility and lipoproteins [26].

Motility and chemotaxis genes have been found conserved among related Campylobacter species with flagella implicated in adhesion, protein secretion, invasion and virulence in pathogenic C. jejuni $[1,27-30]$. Biosynthesis of flagella requires the involvement of more than 40 structural and regulatory proteins including a type III secretion system for flagellar assembly [28,30-32]. The Cff flhA gene based on genome alignments was found to be absent in the available Cfv sequence contigs, and coincided with the ordered alignment gap/non-sequenced section relative to Cff. However, one chemotaxis regulatory protein campy.fasta.screen.Contig1091 orf6 appears to be absent in Cff (Additional file 1). We identified a lower complement of homologues associated with motility in $\operatorname{Cff}(\mathrm{n}=$ 41) compared with the other Campylobacter spp. ( $\mathrm{n}=55-$ 66) $[1]$, however, the analysis of the incomplete Cfv genome identified a higher number of homologues $(\mathrm{n}=$ 46) than the total Cff sequence. PCR assays based on a subset of flagellar genes $(f l g H, f l h F, f l i H, f l h A$ and $f h l B)$, demonstrated conservation of these sequences at least among the members of our panel of $C$. fetus strains including both subspecies (although flhA could not be identified 
Table 2: Reference strains tested in C. fetus PCR assays

\begin{tabular}{lll}
\hline \multicolumn{1}{c}{ Species and subspecies } & \multicolumn{1}{c}{ Strain } & \multicolumn{1}{c}{ Sourcel } \\
\hline C. fetus subsp. venerealis & $98-109383$ (Biovar venerealis) & Field Isolate (DPI\&F, QLD) \\
C. fetus subsp. venerealis & I9438 (Biovar venerealis) & ATCC I9438 \\
C. fetus subsp. venerealis & AZUL-94 (Biovar venerealis) & UNSAM, Argentina \\
C. fetus subsp. venerealis & Biovar venerealis & Pfizer Animal Health \\
C. fetus subsp. venerealis & Biovar intermedius & Pfizer Animal Health \\
C. fetus subsp. fetus & $98-118432$ & Field Isolate (DPI\&F, QLD) \\
C. fetus subsp. fetus & 15296 & ATCC I5296 \\
C. coli & 11353 & NTCC \\
C. jejuni subsp. jejuni & 11168 & NTCC \\
C. hyointestinalis & N3I45 & Field Isolate (DPI\&F, QLD) \\
C. sputorum subsp. bubulus & Y429I-I & Field Isolate (DPI\&F, QLD) \\
Pseudomonas aeruginosa & 27853 & ATCC \\
Proteus vulgaris & 6380 & ATCC \\
Neospora caninum & 50843 & ATCC \\
Tritrichomonas foetus & YVL-W & Field Isolate (DPI\&F, QLD)
\end{tabular}

'Legend: ATCC - American Type Culture Collection; NTCC - National Type Culture Collection; UNSAM - Universidad Nacional de General San Martín; DPI\&F - Department of Primary Industries and Fisheries

in the available Cfv contigs). An additional assay designed to amplify the flaB sequence of the Cfv AZUL-94 strain did not amplify other Cfv biovar venerealis strains but did amplify Cfv intermedius and the Cff isolates. We have not confirmed if this is attributed to flaB sequence variation or an absence of the gene in different geographical $C f v$ biovar venerealis strains, this gene has been targeted however for genotyping studies in other Campylobacter species [33]. This study does confirm that the complete Cfv genome may harbour more flagellar/motility homologues than Cff. Virulent C. jejuni harbours more flagellar genes than less virulent species C. coli, C. lari and C. upsaliensis [1].

Adherence of other Campylobacter species to gut epithelial cells is mediated by multiple adhesins including cadF (Campylobacter adhesion to fibronectin); [34], PEB1 protein (putative binding component of an ABC transporter), [35], JlpA (jejuni lipoprotein A), [36] and a 43-kDa major outer membrane protein [37], confirmed as conserved in C. jejuni, C. lari, C. upsaliensis and C. coli genomes [1]. Cfv homologues for PEB1 and fibronectin-binding (FN-binding) proteins were confirmed with the remaining 3 absent in the genome contigs currently available. However, only the PEB1 protein was identified in the complete Cff genome sequence 82-40. Fibronectin is known to enhance $C$. fetus attachment [38] however in the absence of an identified C. fetus cadF homologue, it appears that the adherence mechanisms in C. fetus may differ from other Campylobacter species. In the case of C. fetus subsp. venerealis, this is perhaps not surprising as $C f v$ colonise the genital tract and not the intestinal tract, thus perhaps novel adhesins will be identified with completion of a $C f v$ genome sequence.
Toxin sequences, two component regulatory systems, plasmids and type IV secretion systems have also been recognised as components in pathogenic Campylobacter spp. [1]. Three cytolethal distending toxin ( $c d t)$ subunits A, B and $\mathrm{C}$ are confirmed as conserved across the four Campylobacter species (C. jejuni, C.lari, C. coli, C. upsaliensis) and C. fetus [22,23]. In addition, the presence of $c d t$ genes is linked to C. jejuni, $C$ coli and C. fetus pathogenesis, where $c d t$ negative strains were found to be less efficient during adherence and invasion in vitro [22,39]. A similar survey of $C$. fetus will assist to confirm if $c d t$ positivity is associated with an increase in pathogenicity. Two-component regulatory (TCR) systems are commonly used by bacteria to respond to specific environmental signals such as temperature [40]. Five TCR systems (pairs of adjacent histidine kinase and response regulator genes) have been identified as conserved across Campylobacter species and confirmed in C. fetus subspecies.

The type IV secretory genes, which are possibly involved in conjugative plasmid transfer or the secretion of virulence factors $[1,18,41]$, were absent in the Cff genome and unique to $C f v$. A large proportion of $C f v$ subspecies specific ORFs (30\%) were harboured in the Cfv contig specific regions. C. upsaliensis and C. jejuni are known to harbour plasmids and evidence does suggest that these plasmids can play a role in pathogenesis. One basic difference between the list of genes absent in Cff and present in Cfv is that many of them are in common to genes present on the plasmids of these related Campylobacter. The type IV secretion system is also found in C. jejuni, C. lari and C. coli plasmid sequence. The unique $C f v$ genome sequences also harboured many phage-like derived genes. The presence of type IV secretion system has also been described 
by Abril et al, 2007 [18], of which the putative VirB6 protein gene was found to be truncated by the insertion element (IScfe1). It is possible that contigs within this $C f v$ unique $80 \mathrm{~Kb}$ suite of contigs represent a number of extrachromosomal DNA plasmids. A wider survey of C. fetus isolates and the presence of plasmids (type IV secretion systems) and phage genes will assist to confirm our observations.

This analysis has provided diagnostic markers to discriminate the Campylobacter subspecies Cfv and Cff, which can be investigated for more general applicability for field use. Most of the Cfv assays based on the incomplete AZUL-94 genome sequence, showed amplification preference for $C f v$ biovar venerealis strains. The Cfv biovar intermedius strains were negative in all but one assay, which was otherwise positive for Cfv AZUL-94 strain only. Curiously, one of the assays designed to Cfv AZUL-94 strain virB9 (type IV Secretion gene) did not amplify other $C f v$ biovar venerealis isolates but did amplify biovar intermedius and the Cff strains tested here. However, as described above the Cff genome sequence (Strain 82-40) does not appear to have type IV secretion genes. A confounding factor in interpreting this data is that different Cff strains may also possess putative plasmid-borne genes and these may potentially be shared between subspecies and $C f v$ biovars. The Cfv AZUL-94 strain could also either consist of a mix of the 2 biovars or represent a novel strain of Cfv. However, assays based on putative plasmid-borne genes have previously demonstrated inconsistencies when applied for subspecies identification in some regions [19]. The parA (plasmid partitioning protein gene), [42] assay target is thought to be plasmid borne, however evidence for plasmids containing parA in $C f v$ has not been confirmed to date $[19,42]$. Very little research has been undertaken to compare the Cfv biovars and the diagnostic targets reported here now need to be further tested in multiple field strains to assess the stability of these markers and therefore the genomic regions in Cfv. However, the results presented do suggest that the $C f v$ research community could benefit from the generation of full genome sequence from both biovars as well as isolates from different geographical continents. Our results also demonstrated putative plasmid sequences are present in $C f v$, absent in Cff, suggesting plasmid profiling and sequencing from $C$. fetus subspecies, biovars and strains will assist to confirm our findings.

\section{Conclusion}

Our assays have highlighted the complexity of virulence factor specificity within C. fetus subspecies and strains probably due to plasmid borne gene elements. We found that most genes important for interactions between a pathogen's surface-exposed proteins and host cells that represent a pivotal step in pathogenesis and virulence were conserved in C. fetus. These genes although impor- tant, did not differentiate the subspecies and therefore not the virulence factors that determined specificity. Instead we found the suite of extrachromosomal type IV secretion system (T4SS) vir genes specific to the Campylobacter fetus subspecies venerealis biovar venerealis AZUL-94 were able to consistently discriminate the C. fetus subspecies fetus in our PCR assays. Complete genomic and plasmid data will ultimately assist to develop definitive tools for comprehensive Campylobacter fetus subspecies differentiation.

\section{Methods}

Bacterial Strains, culture conditions and DNA preparation Campylobacter fetus subsp. venerealis AZUL-94, an Argentinean field strain isolated from a bovine aborted fetus in 1994 was grown routinely on Tryptic Soy Agar plates or in Brain Heart Infusion (BHI) and cultivated under microaerobic conditions in anaerobic jars with CampyGen envelopes (OXOID) at $37^{\circ} \mathrm{C}$. Total DNA from Campylobacter fetus venerealis was isolated by the classical SDS/proteinase K/Phenol/Chloroform extraction method [43]. The Pfizer stains were originally isolated by CSIRO Australia [44].

\section{Library construction, DNA sequencing and assembly}

Genomic DNA was randomly sheared by nebulization, treated with Bal31 nuclease and blunt ended with T4 DNA polymerase. Fragments were size fractionated by agarose gel electrophoresis and ligated to dephosphorylated HincII-digested pBS plasmid. Three libraries with insert size of approximately $2 \mathrm{Kbp}$ (Cf1), $4 \mathrm{Kbp}$ (Cf2), and $6 \mathrm{Kbp}$ (Cf3) were generated. Template preparation and DNA sequencing were performed as described [45] from randomly selected clones. Single-pass sequencing was performed on each template using T7 or T3 primer. Sequencing reads, obtained from the three genomic libraries (Cf1, Cf2, Cf3) were masked against plasmid vector and basecalled with phred (-trim_qual). Those sequences with at least 50 good quality bases after trimming were retained for assembly. After reaching $\sim 4.5 \times$ shotgun coverage, assembly was done using the phredPhrap script provided with phrap. The autofinish functionality of consed was used to select candidate clones for re-sequencing to increase sequence coverage, decrease the number of contigs and increase the consensus quality in a number of cases. Additional information on Campylobacter fetus venerealis sequencing can be found in additional file 6 .

\section{Nucleotide sequence accession numbers}

Sequence data have been deposited in the WGS division of GenBank under the following accession numbers: ACLG01000001... ACLG0101187

\section{Genomic Data}

A subset of $273 \mathrm{Cfv}$ contig sequences (lengths greater than $2 \mathrm{~Kb}$ ) from 1,187 the assembled contigs (Genbank ref nos) was generously supplied by the UNSAM, Argentina 
for this analysis. The assembled contigs have been submitted to GenBank as a part of the WGS division (GenBank:

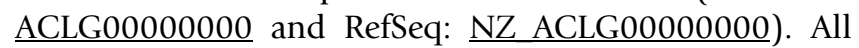
manuscript referenced contig ORFs are listed in the Additional files 1 and 2 .

Completed Campylobacter genomic sequences were obtained from NCBI RefSeq Genome http:// www.ncbi.nlm.nih.gov. All genomic detail for Campylobacter species listed was downloaded from NCBI http:// www.ncbi.nlm.nih.gov/Genomes/ genome division, $28^{\text {th }}$ April, 2008. Campylobacter species included $C$ concisus 13826, C. curvus 525.92, C. fetus subsp. fetus 82-40, C. hominis ATCC BAA-381, C. jejuni RM1221, C. jejuni subsp. doylei 269.97, C. jejuni subsp. jejuni 81-176 and C. jejuni subsp. jejuni 81116.

Alignment of Campylobacter genomes was conducted using BLAT [46] 90 percent identity. The BLAT results were then filtered for a minium $50 \%$ alignment. The two $C$. fetus subspecies were then displayed in Argo [47] (Figure $1)$.

\section{Alignment of genomic Cfv Contigs based on Cff}

The 273 Cfv AZUL-94 contigs were aligned to the Cff 8240 genome (NC_008599) using BLAT [46] (>90\% identity). $C f v$ contigs were ordered and assembled based on the best BLAT alignments between Cfv and Cff based on Cff position and strand orientations into a contiguous pseudomolecule. Unaligned contigs were concatenated to the pseudomolecule linear sequence.

\section{Cfv Open Reading Frame Identification \& Annotation} ORF prediction was conducted on the $273 \mathrm{Cfv}$ using Glimmer3 [48] for ORF lengths greater than 100 nucleotide bases resulting in 1474 open reading frames (ORF). The $273 C f v$ and 1474 ORF were subsequently screened against public NCBI protein (nr, patent), String [49], COG [50], and NCBI Conserved Domain databases with the BLAST program [40]. These results were then categorised using BIOPERL [51] scripts based on alignment percent identity (PID) and query coverage to provide the following six alignment categories, (1) known protein > $80 \%$ PID and $>80 \%$ query coverage, (2) known protein $>$ $30 \%$ PID and $>80 \%$ query coverage, (3) hypothetical protein $>80 \%$ PID and $>80 \%$ query coverage (4) hypothetical protein $>30 \%$ PID and $>80 \%$ query coverage, (5) alignments with an expected value less than $1 e-05,<30 \%$ PID and $<80 \%$ query coverage, and (6) alignments greater than 1 e- $05<30 \%$ PID,$<80 \%$ query coverage.

\section{Campylobacter protein similarity to Cfv ORF}

Campylobacter complete proteome sequence and protein detail were downloaded from NCBI http:// www.ncbi.nlm.nih.gov/genomes/lproks.cgi. The 8 complete campylobacter proteome sets were compared to our Cfv ORF set using BlastMatrix [20] at an ARL 0.75 and an e-value $<1$ e-05 (results in additional file 4).

\section{Putative Virulence Genes}

The functional categories for $C f v$ ORFs were determined based on the String Database [49] categories developed on NCBI COG database role descriptions. The main categories being Cellular processes and signaling, Information storage and processing, Metabolism, Poorly characterized, No mapping, Non Orthologous Group (NOG) and KOG (euKaryote Orthologous Group). The ORFs identified in $C f v$ were screened against the String database and alignment results were filtered using Bioperl for greater than $80 \%$ query coverage and 30\% PID or with an expected value $<1 \mathrm{e}-05$. These ORFs were then screened against NCBI protein database to determine selected putative virulence gene representation in the Campylobacter genus.

\section{Primer Design}

Primer sets were designed on $C f v$ putative virulence genes and genes unique to $C f v$ using Primer3 [52] (Additional file 3: Table S3). Primers were screened against the Cfv AZUL-94 strain and Cff (strain 82-40) genome data and public databases to confirm specificity. Assays were conducted in $20 \mu \mathrm{l}$ reaction volumes, using $10 \mathrm{nM}$ of each forward and reverse primer (Additional file 3: Table S3), $1 \times$ PCR reaction buffer with $25 \mathrm{mM} \mathrm{Mg}^{2+}$ (HotMaster Taq buffer, Eppendorf, Germany), $200 \mu \mathrm{M}$ dNTPs, 1 U Hotmaster $^{\mathrm{TM}}$ Taq DNA polymerase and 1 ng of C. fetus DNA. The reactions were cycled in a Gradient Palm Cycler (Corbett Research, Australia), using the following temperature profile: an initial denaturation at $94^{\circ} \mathrm{C}$ for $2 \mathrm{~min}$, followed by 35 cycles of denaturation at $94^{\circ} \mathrm{C}$ for $20 \mathrm{~s}$, annealing at 45 to $57^{\circ} \mathrm{C}$ (dependent on primer pair, Additional file 3: Table S3) for $10 \mathrm{~s}$, and extension at $72^{\circ} \mathrm{C}$ for 30s including a final single extension for $7 \mathrm{~min}$ at the end of the profile. Amplification products were separated in 2\% TBE ( $89 \mathrm{mM}$ Tris borate, $2 \mathrm{mM}$ EDTA, $\mathrm{pH}$ 8) agarose gels using 100 bp ladder (Invitrogen) and were visualised under UV illumination by ethidium bromide staining. DNA preparations from strains were screened in all assays (Table 2).

\section{Authors' contributions}

PM conducted the bioinformatics analysis and the drafting of the manuscript. AL made substantial contributions to the manuscripts conception and design, the acquisition, analysis and interpretation of the data and the drafting of the manuscript. WB carried out the molecular analysis. DS, FA, DC and RU were responsible for the sequencing and assembly of $C f v$ and provided final approval of the manuscript version to be published. RA 
and $\mathrm{MB}$ made substantial contribution to data interpretation, drafting the manuscript and its critical revision.

\section{Additional material}

\section{Additional File 1}

List of C. fetus subsp. venerealis specific ORF and ORF protein analyses record. The data provided represent the Blast analysis of $\mathrm{C}$. fetus subsp. venerealis specific ORF against protein dataset. Table lists contig ORF, ORF contig position, protein accession, protein description, expected value of orf alignment to the protein sequence and percentage identities in the alignment.

Click here for file

[http://www.biomedcentral.com/content/supplementary/14712180-9-86-S1.xls]

\section{Additional File 2}

List of $\mathrm{C}$. fetus virulence gene contigs targeted in PCR assays. The data provided represent the Blast analysis of $\mathrm{C}$. fetus subsp. venerealis specific ORF against protein dataset. Table lists contig ORF, ORF contig position, protein accession, protein description, expected value of orf alignment to the protein sequence and percentage identities in the alignment.

Click here for file

[http://www.biomedcentral.com/content/supplementary/1471-

2180-9-86-S2.xls]

\section{Additional File 3}

Supplemental Tables. Table S1, Table S2 and Table S3.

Click here for file

[http://www.biomedcentral.com/content/supplementary/1471-

2180-9-86-S3.doc]

\section{Additional File 4}

Campylobacter proteome matrix analysis. An alignment Matrix displays protein similarity between the available Campylobacter complete proteomes (protein) and Cfv ORF (translated to amino acid). Percentage gene duplication is displayed as a percentage and as a heat map within species and across species and stains.

Click here for file

[http://www.biomedcentral.com/content/supplementary/14712180-9-86-S4.png]

\section{Additional File 5}

Plasmid pCFV108 protein alignment to Campylobacter fetus venerealis ORFs. Diagram shows Plasmid pCFV108 and AZUL-94 Contig1185 ORF homology, Campylobacter homology is shaded in pink. Contig1185.orf00004 aligns to MobA (ABK41363) and Contig1185.orf00007 aligns to RepE (ABK41364).

Click here for file

[http://www.biomedcentral.com/content/supplementary/14712180-9-86-S5.png]

\section{Additional File 6}

Campylobacter fetus venerealis genome sequencing and assembly data. Campylobacter fetus venerealis genome sequencing and assembly information.

Click here for file

[http://www.biomedcentral.com/content/supplementary/14712180-9-86-S6.doc]

\section{Acknowledgements}

We thank Diego Rey Serantes, Fernanda Peri and Rodrigo Pavón for technical assistance. The Azul94 strain of $\mathrm{Cfv}$ was a kind gift of Biogenesis S.A. This work was partially supported by grants from the World Bank/UNDP/ WHO Special Program for Research and Training in Tropical Diseases (TDR) to D.O.S, and grant PICT 99 0I-06565 from ANPCyT to RAU. F.A., D.J.C., R.A.U., and D.O.S. are members of the Research Career of the CONICET, Buenos Aires, Argentina.

We wish to acknowledge funds from Meat \& Livestock Australia AHW.036. The authors acknowledge technical support from Ms Catherine Minchin, Ms Bronwyn Venus and Ms Sandra Jarrett. The authors also wish to thank Pfizer Australia for the provision of DNA from the Pfizer strains of $C$. fetus subspecies venerealis biovars and DPI\&F Animal Research Institute culture collection for the use of DPI\&F reference isolates utilised in this study.

\section{References}

I. Fouts DE, Mongodin EF, Mandrell RE, Miller WG, Rasko DA, Ravel J, Brinkac LM, DeBoy RT, Parker CT, Daugherty SC: Major structural differences and novel potential virulence mechanisms from the genomes of multiple Campylobacter species. PLoS Biol 2005, 3(I): 15 .

2. Garcia MM, Eaglesome MD, Rigby C: Campylobacters important in veterinary medicine. Vet Bull 1983, 53:793-818.

3. Mshelia GD, Singh J, Amin JD, Woldehiwet Z, Egwu GO, Murray RD: Bovine venereal campylobacteriosis: an overview. $C A B$ Reviews: Perspectives in Agriculture, Veterinary Science, Nutrition and Natural Resources 2007, 2(80): 14.

4. McMillen L, Fordyce G, Doogan VJ, Lew AE: Comparison of Culture and a Novel 5' Taq Nuclease Assay for Direct Detection of Campylobacter fetus subsp. venerealis in Clinical Specimens from Cattle. J Clin Microbiol 2006, 44:938-945.

5. Parkhill J: The genome sequence of the food-borne pathogen Campylobacter jejuni reveals hypervariable sequences. Nature 2000, 403(6770):665-668.

6. On SL, Harrington CS: Evaluation of numerical analysis of PFGE-DNA profiles for differentiating Campylobacter fetus subspecies by comparison with phenotypic, PCR and I6S rDNA sequencing methods. J Appl Microbiol 200I, 90(2):285-293.

7. Leece JG: Some biochemical characteristics of Vibrio fetus and other related Vibrios isolated from animals. J Bacteriol 1958, 76:312-316

8. Clark BL, Dufty JH, Monsbourgh MJ: A method for maintaining the viability of Vibrio fetus var. venerealis in samples of preputial secretions collected from carrier bulls. Aust Vet $J 1972$, 48(8):462-464.

9. Clark BL, Dufty JH: Isolation of Campylobacter fetus from bulls. Aust Vet J 1978, 54:262-263.

10. Jones RL, Davis MA, Vonbyern H: Cultural procedures for the isolation of Campylobacter fetus subsp. venerealis from preputial secretions and the occurrence of antimicrobial resistance. Proceedings of the Annual Meeting of the American Association of Veterinary Laboratory Diagnosticians 1985, 28:225-238.

II. van Bergen MA, Simons G, Graaf-van Bloois $L$ van der, van Putten JP, Rombout J, Wesley I, Wagenaar JA: Amplified fragment length polymorphism based identification of genetic markers and novel PCR assay for differentiation of Campylobacter fetus subspecies. J Med Microbiol 2005, 54:12 I7-I224.

12. Chang W, Ogg JE: Transduction in Vibrio fetus. Am J Vet Res 1970, 31:919-924.

13. Chang W, Ogg JE: Transduction and mutation to glycine tolerance in Vibrio fetus. Am J Vet Res 1971, 32:649-653.

14. Veron M, Chatelain R: Taxonomic Study of the genus Campylobacter Sebald and Veron and designation of the neotype strain for the type species. Campylobacter fetus (Smith and Taylor) Sebald and Veron. Int J Sys Bacteriol 1973, 23:122-134.

15. van Bergen MA, Dingle KE, Maiden MC, Newell DG, Graaf-Van Bloois $L$ van der, van Putten JP, Wagenaar JA: Clonal nature of Campylobacter fetus as defined by multilocus sequence typing. J Clin Microbiol 2005, 43:5888-5898. 
16. Schulze F, Bagon A, Muller W, Hotzel H: Identification of Campylobacter fetus subspecies by phenotypic differentiation and PCR. J Clin Microbiol 2006, 44(6):2019-2024.

17. Hum S, Quinn K, Brunner J, On SL: Evaluation of a PCR assay for identification and differentiation of Campylobacter fetus subspecies. Aust Vet J 1997, 75:827-83I.

18. Abril C, Vilei EM, Brodard I, Burnens A, Frey J, Miserez R: Discovery of insertion element ISCfe I: a new tool for Campylobacter fetus subspecies differentiation. Clin Microbiol Infect 2007 13(10):993-1000.

19. Willoughby K, Nettleton PF, Quirie M, Maley MA, Foster G, Toszeghy $M$, Newell DG: A multiplex polymerase chain reaction to detect and differentiate Campylobacter fetus subspecies fetus and Campylobacter fetus-species venerealis: use on UK isolates of $C$. fetus and other Campylobacter spp. J Appl Microbiol 2005, 99(4):758-766.

20. Binnewies TT, Hallin PF, Staerfeldt HH, Ussery DW: Genome Update: proteome comparisons. Microbiology 2005, I 5 I ( $\mathbf{P t}$ I): I-4.

21. Kienesberger S, Gorkiewicz G, Joainig MM, Scheicher SR, Leitner E, Zechner EL: Development of Experimental Genetic Tools for Campylobacter fetus. Appl Environ Microbiol 2007 73(14):4619-4630.

22. Asakura M, Samosornsuk W, M T, Kobayashi K, Misawa N, Kusumoto M, Nishimura K, Matsuhisa A, Yamasaki S: Comparative analysis of cytolethal distending toxin (cdt) genes among Campylobacter jejuni, C. coli and C. fetus strains. Microb Pathog 2007, 42(5-6): I $74-183$

23. Lew AE, Guo S-Y, Venus B, Moolhuijzen P, Sanchez D, Trott D, Burrell $P$, Wlodek $B$, Bellgard $M$ : Comparative genome analysis applied to develop novel PCR assays to characterise and identify Campylobacter fetus subsp. venerealis isolates. Zoonoses and Public Health 2007, 54(Supplement I): I 54.

24. Salama SM, Garcia MM, Taylor DE: Differentiation of the subspecies of Campylobacter fetus by genomic sizing. Int J Sys Bacteriol 1992, 42:446-450.

25. Hiett KL, Stintzi A, Andacht TM, Kuntz RL, Seal BS: Genomic differ ences between Campylobacter jejuni isolates identify surface membrane and flagellar function gene products potentially important for colonizing the chicken intestine. Funct Integr Genomics 2008, 8:407-420.

26. Vivona S, Gardy II, Ramachandran S, Brinkman F, Raghava GPS, Flwer DR, Filippini F: Computer-aided biotechnology form immunoinformatics to reverse vaccinology. Trends in biotechnol 2007 26(4): 190-200.

27. Wassenaar TM, Bleumink-Pluym NM, Zeijst BA van der: Inactivation of Campylobacter jejuni flagellin genes by homologous recombination demonstrates that flaA but not flaB is required for invasion. EMBO J 1991, 10:2055-206I.

28. Carrillo CD, Taboada E, Nash JHE, Lanthier P, Kelly J, Lau PC, Verhulp $R$, Mykytczuk O, Sy J, Findlay WA: Genome-wide expression analyses of Campylobacter jejuni NCTCI I I 68 reveals coordinate regulation of motility and virulence by flhA. J Biol Chem 2004, 279( I 9):20327-20338.

29. Yao R, Burr DH, Doig P, Trust TJ, Niu H: Isolation of motile and non-motile insertional elements of Campylobacter jejuni: The role of motility in adherence and invasion of eukaryotic cells. Mol Microbiol 1994, I 4:883-893.

30. Fernando $U$, Biswas $D$, Allan B, Willson $P$, Potter AA: Influence of Campylobacter jejuni fliA, rpoN and flgK genes on colonization of the chicken gut. Int I Food Microbiol 2007, I I 8: | 94-200.

31. Konkel ME, Klena JD, River-Amill V, Monteville MR, Biswas D, Raphael $B$, Mickelson J: Secretion of virulence proteins from Campylobacter jejuni is dependent on a functional flagellar export apparatus. J Bacteriol 2004, 186:3296-3303.

32. Jagannathan A, Constantinidou C, Penn CW: Roles of rpoN, fliA and flgR in expression of flagella in Campylobacter jejuni. J Bacteriol 2001, I 83:2937-2942.

33. Mellmann A, Mosters J, Bartelt E, Roggentin P, Ammon A, Friedrich AW, Karch H, Harmsen D: Sequence-based typing of flaB is a more stable screening tool that typing of flaA for monitoring of Campylobacter populations. I Clin Microbiol 2004, 42:4840-4842

34. Konkel ME, Garvis SG, Tipton SL, Anderson DE, Cieplak W: Identification and molecular cloning of a gene encoding a fibronec- tin-binding protein (CadF) from Campylobacter jejuni. Mol Microbiol 1997, 24:953-963.

35. Pei Z, Burucoa C, Grignon B, Baqar S, Huang X-Z, Kopecko DJ, Bourgeois AL, Fauchere J-L, Blaser MJ: Mutation in the peb IA locus of Campylobacter jejuni reduces interactions with epithelial cells and intestinal colonization of mice. Infect Immun 1998 66:938-943.

36. Jin S, Joe A, Lynett J, Hani EK, Sherman P, Chan VL: JIPA, a novel surface-exposed lipoprotein specific to Campylobacter jejuni, mediates adherence to host epithelial cells. Mol Microbiol 200 I, 39:1225-1236.

37. Moser I, Schroeder W, Salnikow J: Campylobacter jejuni major outer membrane protein and a 59-kDa protein are involved in binding to fibronectin and INT $\mathbf{4 0 7}$ cell membranes. FEMS Microbiol Letts 1997, I 57:233-238.

38. Graham LL, Friel T, Woodman RL: Fibronectin enhances Campylobacter fetus interaction with extracellular matrix components and INT 407 cells. Can J Microbiol 2008, 54:37-47.

39. Jain K, Prasad KN, Sinha S, Husain N: Differences in virulence attributes between cytolethal distending toxin positive and negative Campylobacter jejuni strains. J Med Microbiol 2008, 57:267-272.

40. Bras AM, Chatteriee S, Wren BW, Newell DG, Ketley JM: A novel Campylobacter jejuni two-component regulatory system important for temperature-dependent growth and colonization. J bacteriol 1999, | 8 I:3298-3302.

4I. Christie PJ, Atmakuri K, Krishnamoorthy V, Jakubowski S, Cascales E: Biogenesis, architecture and function of bacterial Type IV secretion systems. Annu Rev Microbiol 2005, 59:45I-485.

42. Ebersbach G, Gerdes K: Plasmid segregation mechanisms. Annu Rev Genet 2005, 39:453-479.

43. Sambrook J, Fritsch EF, Maniatis T: In Molecular cloning: A laboratory manual. Cold Spring Harbor, NY: Cold Spring Harbor Laboratory Press; 1989.

44. Clark BL, Dufty JH, Monsbourgh MJ, Parsonson IM: Immunisation against bovine vibriosis due to Campylobacter fetus subsp. fetus biotype intermedius. Aust Vet J 1976, 52:362-365

45. Agüero $F$, Verdún RE, Frasch $A C$, Sánchez $D O$ : A random sequencing approach for the analysis of the Trypanosoma cruzi genome: general structure, large gene and repetitive DNA families, and gene discovery. Genome Res 2000, I O(I 2): I996-2005

46. Kent W]: BLAT-The BLAST-Like Alignment Tool. Genome Res 2002, I 2(4):656-664

47. Engels R, Yu T, Burge C, Mesirov JP, DeCaprio D, Galagan JE: Combo: a whole genome comparative browser. Bioinformatics 2006, 22(14): I782-I783.

48. Delcher AL, Harmon D, Kasif S, White O, Salzberg SL: Improved microbial gene identification with GLIMMER. Nucleic Acids Research 1999, 27(23):4636-464I

49. von Mering C, Jensen JI, Kuhn M, Chaffron S, Doerks T, Kruger B, Snel $B$, Bork P: STRING 7-recent developments in the integration and prediction of protein interactions. Nucleic Acids Res 2007:D358-D362.

50. Tatusov RL, Fedorova ND, Jackson JD, Jacobs AR, Kiryutin B, Koonin EV, Krylov DM, Mazumder R, Mekhedov SL, Nikolskaya AN: The COG database: an updated version includes eukaryotes. BMC Bioinformatics 2003, 4(4I):.

5I. Stajich JE, Block D, Boulez K, Brenner SE, Chervitz SA, Dagdigian C Fuellen G, Gilbert JG, Korf I, Lapp H, et al.: The Bioperl toolkit: Perl modules for the life sciences. Genome Res 2002 I2(10): $1611-1618$

52. Rozen S, Skaletsky H: Primer3 on the WWW for general users and for biologist programmers. Methods Mol Biol 2000, 132:365-386. 